\title{
Effects of Trauma Center Establishment on the Clinical Characteristics and Outcomes of Patients with Traumatic Brain Injury : A Retrospective Analysis from a Single Trauma Center in Korea
}

\author{
Jang Soo Kim, M.D., ${ }^{1}$ Sung Woo Jeong, A.A., ${ }^{1}$ Hyo Jin Ahn, R.N., ${ }^{2}$ Hyun Ju Hwang, R.N., ${ }^{2}$ Kyu-Hyouck Kyoung, M.D., \\ Soon Chan Kwon, M.D., 'Min Soo Kim, M.D., \\ Department of Neurosurgery, Ulsan University Hospital, University of Ulsan College of Medicine, Ulsan, Korea \\ Trauma center, ${ }^{2}$ Ulsan University Hospital, University of Ulsan College of Medicine, Ulsan, Korea
}

Objective : To investigate the effects of trauma center establishment on the clinical characteristics and outcomes of trauma patients with traumatic brain injury (TBI).

Methods : We enrolled 322 patients with severe trauma and TBI from January 2015 to December 2016. Clinical factors, indexes, and outcomes were compared before and after trauma center establishment (September 2015). The outcome was the Glasgow outcome scale classification at 3 months post-trauma.

Results : Of the 322 patients, 120 (37.3\%) and 202 (62.7\%) were admitted before and after trauma center establishment, respectively. The two groups were significantly different in age $(p=0.038)$, the trauma location within the city $(p=0.010)$, the proportion of intensive care unit (ICU) admissions $(p=0.001)$, and the emergency room stay time $(p<0.001)$. Mortality occurred in 37 patients (11.5\%). Although the preventable death rate decreased from before to after center establishment ( $23.1 \%$ vs. $12.5 \%)$, the difference was not significant. None of the clinical factors, indexes, or outcomes were different from before to after center establishment for patients with severe TBI (Glasgow coma scale score $\leq 8$ ). However, the proportion of inter-hospital transfers increased and the time to emergency room arrival was longer in both the entire cohort and patients with severe TBI after versus before trauma center establishment.

Conclusion : We confirmed that for patients with severe trauma and TBI, establishing a trauma center increased the proportion of ICU admissions and decreased the emergency room stay time and preventable death rate. However, management strategies for handling the high proportion of inter-hospital transfers and long times to emergency room arrival will be necessary.

Key Words : Trauma centers · Brain injuries, Traumatic · Glasgow coma scale · Glasgow outcome scale · Mortality.

- Received : February 12, 2018 •Revised : April 17, 2018 •Accepted : April 30, 2018

- Address for reprints : Min Soo Kim, M.D.

Department of Neurosurgery, Ulsan University Hospital, University of Ulsan College of Medicine, 877 Bangeojinsunhwando-ro, Dong-gu, Ulsan 44033, Korea Tel : +82-52-250-7139, Fax : +82-52-250-7138, E-mail : kist1817@gmail.com

This is an Open Access article distributed under the terms of the Creative Commons Attribution Non-Commercial License (http://creativecommons.org/licenses/by-nc/4.0) which permits unrestricted non-commercial use, distribution, and reproduction in any medium, provided the original work is properly cited. 


\section{INTRODUCTION}

A patient with severe trauma is an individual with serious or major complications such as hemorrhagic shock or multiple organ dysfunctions, who is suffering from major organ damage or extensive damage to other body parts due to trauma, such as swelling or penetration ${ }^{8,28}$. The speed of treatment after an accident in such patients plays a large role in their prog-

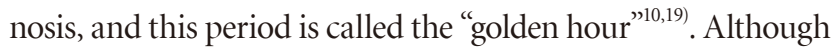
a sole injury may occur, multiple injuries are more common and may negatively affect the patient's prognosis ${ }^{3,7)}$. Therefore, a multi-disciplinary approach to multiple injuries is needed in patients with severe trauma ${ }^{1)}$.

Notably, the preventable death rate from severe trauma in Korea is $>30 \%$, which is three times higher than the mortality rate of $10 \%$ in other developed countries ${ }^{14,15)}$. Accordingly, in 2012, the Korean government began assigning and operating trauma centers that provide rapid and multi-disciplinary access to treatment to reduce the mortality rate from severe trauma ${ }^{5,27,29)}$. Indeed, the Korean government stated that new centers would be opened every few years, and provided 8 billion KRW before trauma center establishment and 2.3 billion KRW annually after trauma center establishment ${ }^{29)}$. With this government support, special facilities for patients with trauma (intensive care units [ICUs], wards, operating rooms, and treatment rooms), equipment (diagnostic imaging equipment, treatment equipment), and trauma specialists have been provided to hospitals throughout the country. These funds also paid for emergency medical services (EMS) related to the medical treatment, research, and management of patients with trauma, as well as to the standard development and training of related staff. Therefore, these functions are supervised and evaluated several times each year by the Korean government ${ }^{21}$.

Among patients with severe trauma, head trauma is the most common and is associated with a high mortality rate ${ }^{6}$. In addition, most patients with traumatic brain injury (TBI) incur significant costs owing to the need for long-term treatment associated with TBI-related complications ${ }^{11,22)}$. To prevent the deterioration of patients with TBI and to improve their prognosis, rapid diagnosis and treatment are essential ${ }^{9}$. Although establishment of the trauma centers, with their rapid and multi-disciplinary approach to treating patients with severe trauma, has improved the prognosis of patients, the mortality rate in Korea has not yet reached the level of other countries with existing trauma centers ${ }^{21)}$. Moreover, the changes in the clinical factors and prognosis of patients with TBI, which account for the largest proportion of patients with severe trauma, following the introduction of the trauma centers, have not been evaluated.

Therefore, the purpose of this study was to investigate the changes in clinical characteristics and outcomes of patients with severe trauma and TBI that occurred from before to after the establishment of a single trauma center in Korea.

\section{MATERIALS AND METHODS}

This study was approved by Ulsan University Hospital (IRB No. 2018-02-013) and the requirement for informed consent was waived.

\section{Patients}

This retrospective study included 21765 patients who visited a single center from January 2015 to December 2016. The inclusion criteria were patients who visited the emergency room with an Injury severity score (ISS) of 15 or more, patients who were admitted to the neurosurgery department mainly due to head injury (patients whose largest ISS was for the head), and patients who were followed up for $>3$ months ${ }^{20)}$. Among the 835 patients with severe trauma, we excluded 303 patients without TBI who were admitted to the department of trauma surgery, 104 patients who were admitted to the department of trauma surgery due to poly-trauma with TBI, 86 patients who were admitted to the department of neurosurgery due to spine trauma, and 20 patients with TBI who died in the emergency room. Ultimately, a total of 322 patients were enrolled in this study.

\section{Trauma center}

Our center is the only tertiary hospital in charge of the final transfer and treatment of patients with severe trauma in the 60-km sector. Many industrial disasters such as explosions occur in this area due to the large number of nearby petrochemical, mechanical, and shipbuilding complexes (Fig. 1). For this reason, the center was established in September 2015 after being selected as the future site of a trauma center in July 2013. Thanks to the support of the Korean government, the trauma 


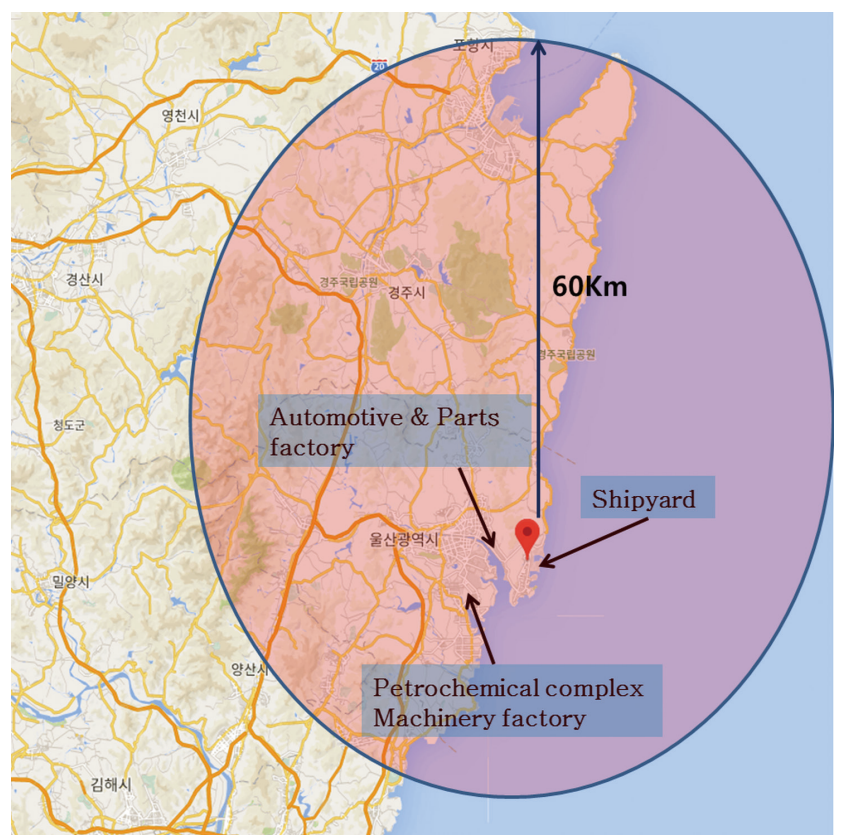

Fig. 1. Location characteristics and responsibility area of the trauma center (black spot : trauma center, black circle : responsibility area).

center consists of ICUs and wards that are dedicated to patients with trauma; trainee doctors, nurses, and trauma-specialized instruments were also provided. Furthermore, the trauma center is supervised annually by the government ${ }^{211}$. Our trauma team consists of staff from the trauma surgery, emergency medicine, orthopedic surgery, and neurosurgery departments. Our department of neurosurgery consists of six neurosurgeons. Physicians selected treatment plans according to their discretion when patients with severe trauma arrived in the emergency room.

\section{Clinical data collection}

Patients' clinical factors were obtained via retrospective chart reviews and included sex, age, trauma mechanism, trauma location, time to emergency room arrival, inter-hospital transfer, admission to the ICU, emergency room stay time, Glasgow coma scale (GCS) score, ISS, Revised trauma score (RTS), and Trauma and injury severity score (TRISS) ${ }^{2,425,26)}$. The trauma mechanism was classified as a car traffic accident (TA), motorcycle TA, bicycle TA, pedestrian TA, cultivator accident, fall, slip, head collision, or unknown mechanism, depending on the cause of the traumatic incident. The trauma location was defined as either within or outside the city at the time of the incident. The time to emergency room arrival was defined as the duration from accident occurrence to arrival in the emergency room. Inter-hospital transfer was defined as a patient who was transferred from another hospital. Admission to the ICU included the trauma ICU and other ICU admissions. The emergency room stay time was defined as the duration from entry in the emergency room to hospitalization in the ward or ICU. The GCS, ISS, RTS, and TRISS measured the patient's initial status in the emergency room. Severe TBI was defined as a patient with a GCS score $\leq 8^{23)}$. We also performed a subgroup analysis of the patients who arrived in the emergency room within 24 hours of the trauma.

\section{Outcome}

The outcome was based on the Glasgow outcome scale (GOS) classification at 3 months after the traumatic event ${ }^{12}$. When mortality occurred before 3 months, this was described as mortality at 3 months. The preventable death rate was also investigated and defined as (preventable death/total death) $\times$ $100^{17)}$.

\section{Statistics}

Statistical analyses were performed using SPSS version 21.0 (IBM Corp., Armonk, NY, USA). Categorical variables were analyzed with chi-squared tests, Fisher's exact tests, or linearby-linear tests, as appropriate. Continuous variables were analyzed with Student's t-tests or Mann-Whitney U tests according to the results of the normality test. The relationship between the GOS and the various indexes (GCS, ISS, RTS, or TRISS) was analyzed by Pearson's correlation analyses. To determine associations with mortality, a logistic regression analysis was performed on variables with a $p$ value $<0.05$. In all analyses, statistical significance was set at $p \leq 0.05$.

\section{RESULTS}

The baseline characteristics of the patients are shown in Table 1. Of the 322 patients, 233 (72.4\%) were male and the mean age was $54.26 \pm 21.37$ years. As for the trauma mechanism, slipping $(\mathrm{n}=108,33.5 \%)$ was the most common cause of trauma, while cultivator accidents $(n=2,0.6 \%)$ were the least common. The most common form of TA was pedestrian $(\mathrm{n}=38,11.8 \%)$, and most of the trauma incidents occurred within the city $(n=287,89.1 \%)$. The mean emergency room stay time was $306.87 \pm 416.02$ minutes. The mean GCS score, 
Korean Trauma Center I Kim JS, et al.

Table 1. In patients with severe trauma and traumatic brain injury, the comparisons between the entire cohort and the subgroup of patients who died

\begin{tabular}{|c|c|c|c|}
\hline & Total $(n=322)$ & Mortality $(n=37,11.5 \%)$ & $p$-value \\
\hline Sex (male) & $233(72.4)$ & $31(83.8)$ & 0.099 \\
\hline Age (years) & $54.26 \pm 21.37$ & $58.46 \pm 15.34$ & 0.100 \\
\hline Trauma mechanism & & & 0.168 \\
\hline Cars TA & $18(6.5)$ & $1(2.7)$ & \\
\hline Motorcycle TA & $34(10.6)$ & $2(5.4)$ & \\
\hline Bicycle TA & $18(5.6)$ & $3(8.1)$ & \\
\hline Pedestrian TA & $38(11.8)$ & $4(10.8)$ & \\
\hline Cultivator accident & $2(0.6)$ & 0 & \\
\hline Fall & $51(15.8)$ & $6(16.2)$ & \\
\hline Slip & $108(33.5)$ & $13(35.1)$ & \\
\hline Head collision & $37(11.5)$ & $4(10.8)$ & \\
\hline Unknown & $16(5.0)$ & $4(10.8)$ & \\
\hline Trauma location within the city & $287(89.1)$ & $37(100)$ & 0.021 \\
\hline After trauma center establishment & $202(62.7)$ & $24(64.9)$ & 0.858 \\
\hline Inter-hospital transfer & $183(56.8)$ & $21(56.8)$ & 1.000 \\
\hline Admission to the ICU & $267(82.9)$ & $37(100)$ & 0.004 \\
\hline Emergency room stay time (minutes) & $306.87 \pm 416.02$ & $158.27 \pm 99.03$ & $<0.001$ \\
\hline GCS & $12.32 \pm 4.01$ & $7.24 \pm 4.32$ & $<0.001$ \\
\hline ISS & $23.19 \pm 5.60$ & $25.54 \pm 5.35$ & 0.007 \\
\hline RTS & $7.118 \pm 1.486$ & $5.484 \pm 1.814$ & $<0.001$ \\
\hline TRISS & $0.9325 \pm 0.3070$ & $0.8482 \pm 0.2488$ & 0.076 \\
\hline
\end{tabular}

Values are presented as mean \pm standard deviation or number (\%). TA : traffic accident, ICU : intensive care unit, GCS : Glasgow coma scale, ISS : Injury severity score, RTS : revised trauma score, TRISS : Trauma and injury severity score

ISS, RTS, and TRISS at the initial evaluation were 12.32 \pm 4.01 , $23.19 \pm 5.60,7.118 \pm 1.486$, and $0.9325 \pm 0.3070$, respectively.

Mortality occurred in 37 patients (11.5\%) (Table 1). Thirty patients $(81.1 \%)$ died of neurologic problems and seven (18.9\%) died of other medical problems. Comparisons between the entire cohort and the subgroup of patients who died (Table 1) revealed that the trauma location was more frequently within the city $(p=0.021)$, the proportion of ICU admissions was higher $(p=0.004)$, and the emergency room stay time $(p<0.001)$ was significantly shorter in patients who died than in the entire cohort. In addition, the GCS score $(p<0.001)$, ISS $(p=0.007)$, and RTS $(p<0.001)$ of patients who died were worse than were those of the entire cohort. However, no significant difference in the TRISS $(p=0.076)$ was identified between the groups.

In total, $120(37.3 \%)$ and 202 patients (62.7\%) were admitted to the hospital before and after trauma center establishment, respectively (Table 2). The two groups were significantly differ- ent in terms of age $(p=0.038)$, the trauma location $(p=0.010)$, the proportion of ICU admissions ( $p=0.001)$, and the emergency room stay time $(p<0.001)$. Specifically, patients admitted after trauma center establishment were older than were patients admitted before trauma center establishment, and the trauma location was more frequently located within the city after than before trauma center establishment. Moreover, the rate of ICU admission was higher and the emergency room stay time was shorter after than before trauma center establishment. The number of inter-hospital transfer patients increased from before to after trauma center establishment, although the difference was not statistically significant $(p=0.347)$. In addition, the outcomes, including mortality $(p=0.776)$ and the GOS classification at 3 months ( $p=0.341$, Fig. 2 A), were not significantly different from before to after center establishment. However, the preventable death rate decreased slightly, but not significantly, from before to after trauma center establishment $(p=0.643)$. 


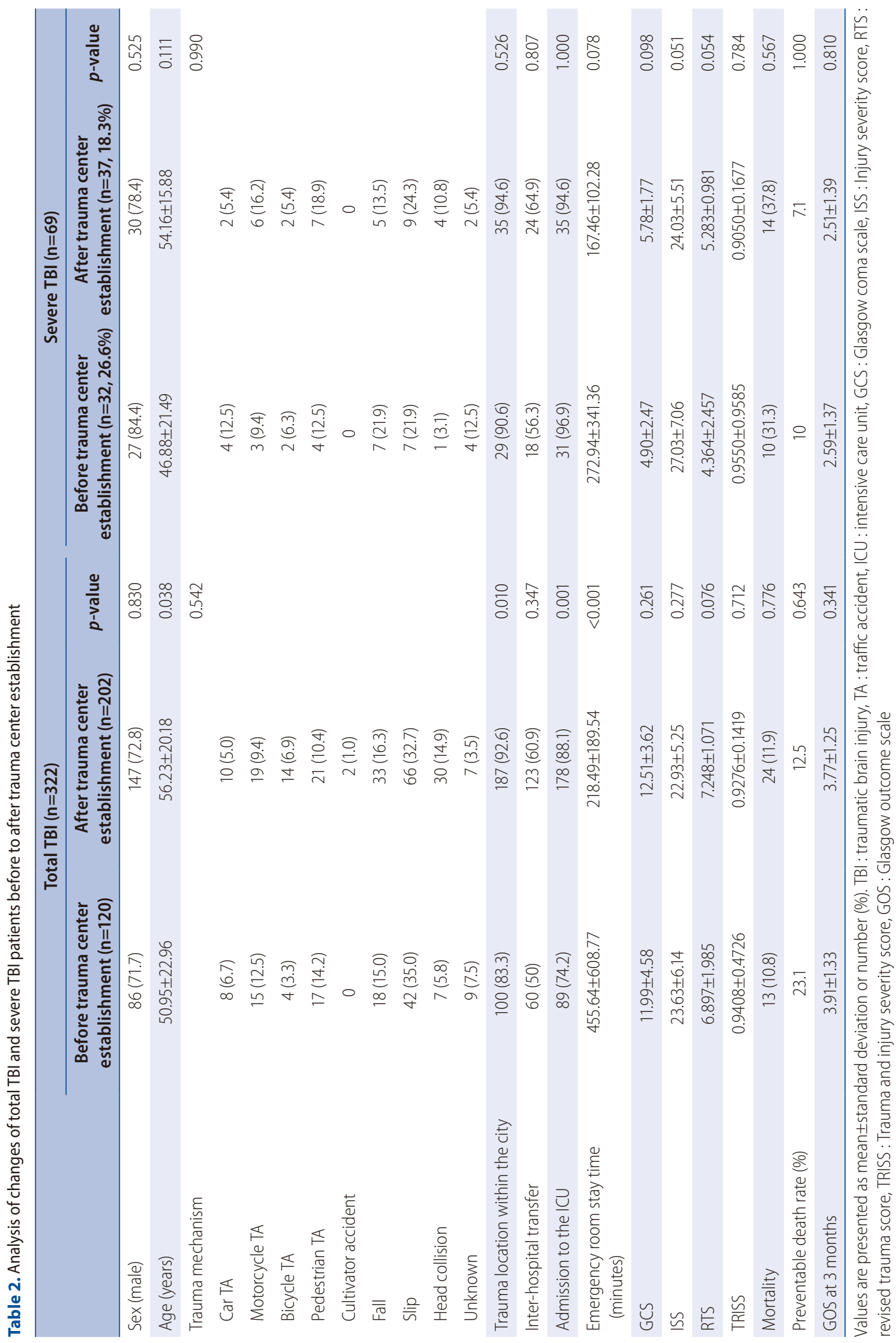




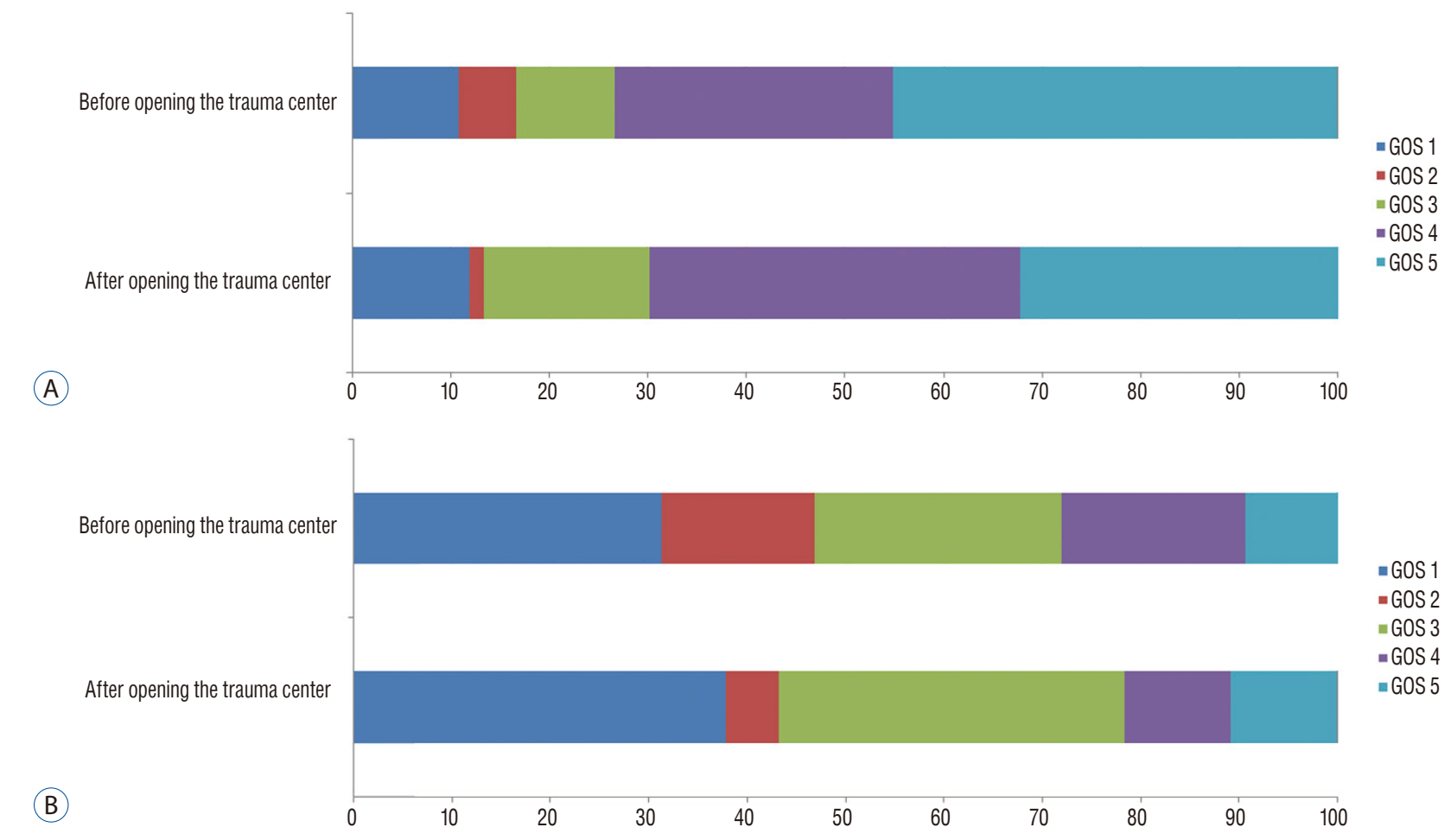

Fig. 2. GOS at 3 months after the accident for all patients (A) and patients with severe traumatic brain injury (B). GOS : Glasgow outcome scale.

When evaluating only those patients with severe TBI (Table 2), we noted that $32(26.6 \%)$ and 37 patients (18.3\%) were admitted before and after trauma center establishment, respectively. However, in contrast to the entire cohort, the age $(p=0.111)$, trauma location within the city $(p=0.526)$, proportion of ICU admissions ( $p=1.000)$, and emergency room stay time ( $p=0.078$ ) were not significantly different from before to after trauma center establishment for patients with severe TBI. Similar to the entire cohort, the number of inter-hospital transfer patients increased slightly, but not significantly, from before to after trauma center establishment $(p=0.807)$. In addition, no differences in outcome, including mortality $(p=0.567)$ and GOS classification at 3 months $(p=0.810$, Fig. $2 B$ ), were identified from before to after trauma center establishment for the patients with severe TBI. Further, like the entire cohort, the preventable death rate decreased slightly, but not significantly, from before to after trauma center establishment for patients with severe TBI $(p=1.000)$.

In our subgroup analysis of those patients who arrived in the emergency room within 24 hours of the traumatic event (Table 3), $86(71.6 \%)$ and 167 patients (82.7\%) were admitted before and after establishment of the trauma center. The two groups were significantly different in terms of the emergency room stay time ( $p=0.003)$, ISS $(p=0.031)$, and TRISS $(p=0.002)$. Specifically, the emergency room stay time was shorter after than before trauma center establishment. However, the ISS and TRISS were more severe before than after trauma center establishment. Interestingly, before center establishment, patients with severe TBI showed a high rate of arriving in the emergency room within 24 hours (87.5\%); after center establishment, this rate remained relatively similar, with only a slight increase (89.2\%). In addition, the time to emergency room arrival was longer after the center was opened than before for both the entire cohort and patients with severe TBI, although the differences were not significant ( $p=0.429$ and 0.304 , respectively).

We also performed a subgroup analysis of inter-hospital transfer patients in entire cohort and found that the mean age of patients who underwent inter-hospital transfer was greater than that of patients who visited the hospital directly (57.02 \pm 18.89 years vs. $51.05 \pm 23.48$ years, $p=0.015$ ). In addition, the mean age before center establishment was not significantly different between patients who underwent inter-hospital transfer and those who visited the hospital directly (53.42 \pm 20.11 years vs. $49.51 \pm 24.13$ years, $p=0.351$ ), but the mean age 


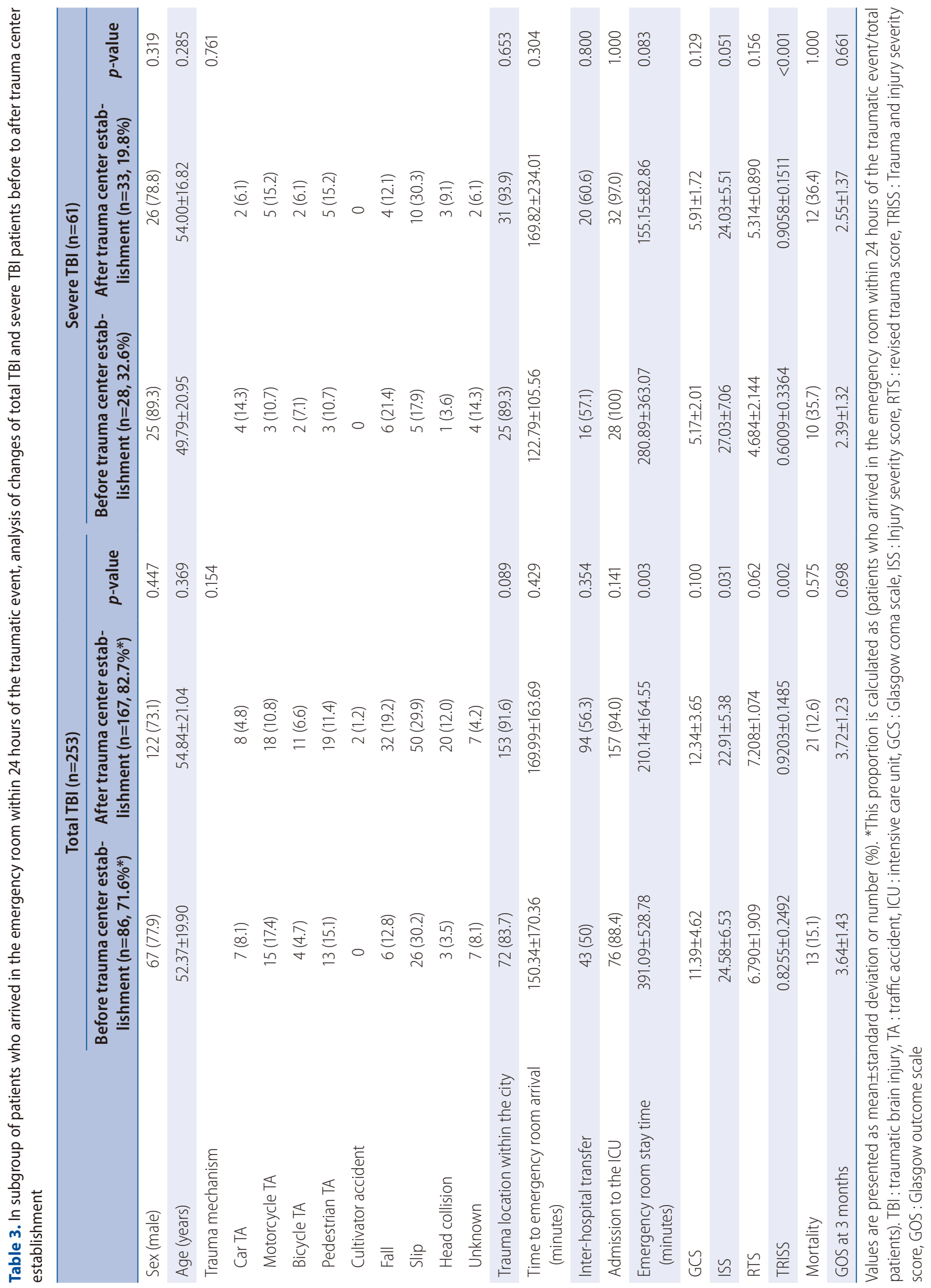


Table 4. Stepwise logistic regression analysis of the initial indexes in emergency room and the mortality

\begin{tabular}{lccc}
\hline & Odds ratio & 95\% confidence interval & $\boldsymbol{p}$-value \\
\hline GCS & 0.709 & $0.607-0.827$ & $<0.001$ \\
ISS & 1.043 & $0.980-1.110$ & 0.190 \\
RTS & 1.175 & $0.830-1.663$ & 0.364 \\
\hline
\end{tabular}

GCS : Glasgow coma scale, ISS : Injury severity score, RTS : revised trauma score

after center establishment was significantly greater for patients who underwent inter-hospital transfer than it was for patients who visited the hospital directly $(58.78 \pm 18.04$ years vs. $52.00 \pm$ 23.15 years, $p=0.024)$. The subgroup analysis of inter-hospital transfer patients who arrived in the emergency room within 24 hours of the accident also revealed that the mean time to emergency room arrival was significantly longer for inter-hospital transfer patients than it was for patients who visited the emergency room directly $(245.45 \pm 205.55$ minutes vs. $65.45 \pm$ 205.55 minutes, $p<0.001)$. In patients with severe TBI, the mean time to emergency room arrival was similarly significantly longer for inter-hospital transfer patients than it was for patients who visited the emergency room directly (211.03 \pm 205.15 minutes vs. $54.28 \pm 91.74$ minutes, $p<0.001)$.

Further analyses revealed that the GCS score $(p<0.001$, $\mathrm{r}=0.597)$, ISS $(p=0.001, \mathrm{r}=-0.191)$, and RTS $(p<0.001, \mathrm{r}=0.487)$, as initial evaluation indexes, were associated with the GOS classification at 3 months post-trauma. However, the TRISS ( $p=0.407, \mathrm{r}=0.047$ ) was not correlated with the GOS classification at 3 months. Notably, the GCS score $(p<0.001)$ was the only indicator correlated with mortality (Table 4).

\section{DISCUSSION}

Since Ulsan University Hospital opened the trauma center on September 17, 2015, we have added 20 beds in the trauma ICU, 40 beds in the trauma ward, two operating rooms, and an angiography room. We also acquired trauma-only computed tomography and X-ray equipment and trauma experts. Here, we found that the mean age of patients increased from before to after center establishment. According to our analyses, this change appears to be driven by an increase in the inter-hospital transfer of elderly patients after center establishment. In addition, we found that after the trauma center opened, the number of patients admitted whose traumatic events occurred within the city increased, likely due to the training of paramedics and medical support personnel and to the announcement of trauma center establishment to the local emergency medical center. However, before the trauma center was established, our hospital had been the only tertiary hospital in the area that was able to manage patients with severe TBI, and thus such patients were already being transferred to our center. In addition, for patients whose severe TBI occurred in a different city, it is likely that the necessary treatment was performed in that city to avoid neurologic deterioration related to the time required for transportation. Therefore, for patients with severe TBI requiring urgent treatment, the proportion of patients with a trauma location within the city was not different from before to after trauma center establishment. Regarding our entire cohort, the present study revealed that after the trauma center was established, the proportion of ICU admissions increased, while the emergency room stay time decreased. These findings may be explained by the increased number of trauma ICU beds and the rapid treatment decisions and admissions that were made after primary surveys were performed by trauma specialists provided by the government. However, since urgent treatment is an important prognostic factor for patients with severe TBI, admission to the ICU and the emergency room stay time were being managed by neurosurgeons prior to the opening of the trauma center. Therefore, the changes in these factors were not statistically significant for patients with severe TBI.

Kane et al. ${ }^{13)}$ previously reported that patients with minor injuries treated at trauma centers most likely did not require trauma services, whereas patients with severe injuries were unlikely to survive regardless of which treatment facility was utilized. Based on our results, we think that the benefits, such as the increased proportion of ICU admissions and decreased emergency room stay times, for patients with moderate or mild TBI were greater than were those for patients with severe TBI after trauma center establishment owing to the active management provided by emergency room trauma specialists. In addition, most patients with mild or moderate TBI tend to show similar courses and outcomes, but patients with severe TBI show varying results depending on the rapidity and effectiveness of treatment. Thus, the outcome of patients with severe TBI may have significantly affected the outcome of the entire cohort. In other words, the lack of change in patient 


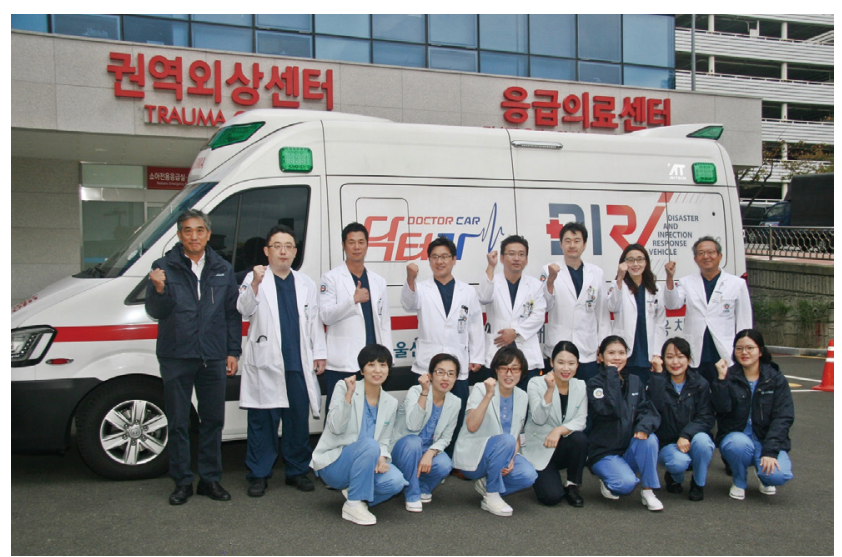

Fig. 3. "Mobile Trauma Unit", dedicated vehicle with doctors for interhospital transfers or direct treatment at the accident site.

outcomes from before to after trauma center establishment may have been due to the lack of significant changes for patients with severe TBI, since such patients were being treated rapidly by the neurosurgeons prior to the opening of the trauma center.

The lack of change in patient outcomes from before to after trauma center establishment may also be due to the lack of change in the number of inter-hospital transfers. Mullins et al. ${ }^{18)}$ reported that the reduced mortality observed throughout the Oregon trauma system was partially attributed to improved training of EMS personnel and to advancements in technology. In addition, he suggested that many tertiary hospitals may be functioning as trauma centers without the benefit of the designation by the Oregon trauma system ${ }^{18}$. A study by Sampalis et al. ${ }^{24)}$ reported a steady increase in the number of severely injured patients that were being transferred to trauma centers post-designation and noted that the overall level of care improved, likely because of more-timely interventions and better access to the resources that trauma centers are provided. In our study, inter-hospital transfers accounted for more than $50 \%$ of patients before center establishment, with this number increasing to $>60 \%$ after the center was opened. In addition, inter-hospital transfer patients had longer emergency room arrival times than did patients who visited the emergency room directly. It is also possible that the inability to take immediate action at the scene of the accident, delays at other hospitals, or the inappropriate action of EMS in interhospital transfers are hampering the improvement of patient outcomes. Therefore, we tried to solve this problem by establishing and operating a dedicated vehicle with doctors, re- ferred to as the "Mobile Trauma Unit," for inter-hospital transfers or direct treatment at the accident site (Fig. 3). Although the effects of this unit on patient outcomes remain unclear because it is early in the trial, we expect to observe beneficial effects in the future.

To reduce the bias for patients with mild or moderate TBI who were hospitalized at other centers and then transferred to our hospital, an analysis of patients who arrived in the emergency room within 24 hours of the accident was conducted. Among the entire cohort, patients were more likely to visit the emergency room within 24 hours after than before center establishment. However, the rate of visiting the emergency room within 24 hours was high for patients with severe TBI both before and after center establishment. Therefore, the accessibility of patients with mild or moderate TBI to the center increased after center establishment. Additionally, we found that the severity indexes were lower after the center was established, likely because of the increased number of patients with mild or moderate TBI due to improved accessibility.

Trauma center establishment was not correlated with mortality. However, although not statistically significant, the preventable death rate decreased after trauma center establishment in both the entire cohort and the severe TBI group. The lack of significance is thought to be due to the small sample size. In addition, our assumption that patients with TBIs occurring outside the city were being treated in that city to avoid neurologic deterioration was confirmed by the fact that all mortality cases had trauma locations within the city. This means that, for patients with severe TBI, the procedure time is important and that other possible centers with severe TBI management may have a policy for rapid treatment regardless of the establishment of the trauma center. Regarding the initial patient status indexes, the only score that was not associated with mortality was the TRISS. Recently, a revised TRISS model has been reported as a replacement for the original TRISS model because of limitations in its ability to evaluate patient survival $^{25)}$. In our study, the TRISS trended towards significance but was not statistically significant due to the limitations of the TRISS model. In addition, it is known that the ISS is one of the most representative indicators of the severity of trauma patients, and our trauma center classifies patients with severe trauma using the ISS. However, for patients with TBI, the consciousness level is an important factor, and this is commonly assessed with the $\mathrm{GCS}^{26)}$. Our data likewise 
showed that the GCS best reflects the outcome and mortality of patients with head injuries.

\section{Limitations}

This study had several limitations. First, our study may have selection bias and compounding effects due to its retrospective nature. Second, this was a small, single-center study. However, our center is the only tertiary hospital in our city and is the only center capable of treating patients with severe trauma. Although our findings may not be generalizable to all trauma patients, our study is thought to be representative of the local patients within this city. Third, we did not consider the effects of patients with multiple traumas, and only the patients who had a head trauma as the main problem and who were admitted to a neurosurgeon from the trauma team were enrolled in this study. In our trauma center, severe patients with poly-trauma are admitted to trauma surgeons, and thus only the patients with head trauma were selected for this study. Fourth, objective classification and evaluation of some factors affecting outcomes was difficult. For example, team activation is controlled uniformly by the government. Regarding the time to the operating room, there were some problems such as the patients who did not undergo surgery was more and the patients who underwent surgery due to deterioration during hospitalization. Fifth, the lack of change in patient outcomes we observed may be due to problems following the initial opening of the trauma center. Mann et al. ${ }^{16)}$ suggested that a trauma system goes through a transitional period that may take up to 10 years to truly stabilize. If the stability of the management process within the center is secured over time, the patients' outcomes may change. Therefore, a long-term study with a large sample size is necessary to address these issues.

\section{CONCLUSION}

In conclusion, we confirmed that for patients with severe trauma and TBI, the rate of ICU admission increased while the emergency room stay time decreased from before to after trauma center establishment. Further, although not statistically significant, the preventive death rate decreased after trauma center establishment. However, despite the establishment of a trauma center, high inter-hospital transfer rates and long emergency room arrival times were still observed. In the future, efforts to reduce these problems will be necessary.

\section{CONFLICTS OF INTEREST}

No potential conflict of interest relevant to this article was reported.

\section{INFORMED CONSENT}

This type of study does not require informed consent.

\section{- Acknowledgements}

This research was supported by Basic Science Research Program through the National Research Foundation of Korea (NRF) funded by the Ministry of Science and ICT (NRF2017R1C1B5018173).

\section{References}

1. Bach JA, Leskovan JJ, Scharschmidt T, Boulger C, Papadimos TJ, Russell $S$, et al. : The right team at the right time - Multidisciplinary approach to multi-trauma patient with orthopedic injuries. Int J Crit IIIn Inj Sci 7 : 32-37, 2017

2. Baker SP, O'Neill B, Haddon W Jr, Long WB : The injury severity score: a method for describing patients with multiple injuries and evaluating emergency care. J Trauma 14 : 187-196, 1974

3. Bonatti H, Calland JF : Trauma. Emerg Med Clin North Am 26 : $625-$ 648, vii, 2008

4. Champion HR, Sacco WJ, Carnazzo AJ, Copes W, Fouty WJ : Trauma score. Crit Care Med 9 : 672-676, 1981

5. Cherry RA, King TS, Carney DE, Bryant P, Cooney RN : Trauma team activation and the impact on mortality. J Trauma 63 : 326-330, 2007

6. Coronado VG, Xu L, Basavaraju SV, McGuire LC, Wald MM, Faul MD, et al. : Surveillance for traumatic brain injury-related deaths--United States, 1997-2007. MMWR Surveill Summ 60 : 1-32, 2011

7. El Mestoui Z, Jalalzadeh H, Giannakopoulos GF, Zuidema WP : Incidence and etiology of mortality in polytrauma patients in a Dutch level I trauma center. Eur J Emerg Med 24 : 49-54, 2017

8. Fröhlich M, Lefering R, Probst C, Paffrath T, Schneider MM, Maegele $M$, et al. : Epidemiology and risk factors of multiple-organ failure after multiple trauma: an analysis of 31,154 patients from the TraumaRegister DGU. J Trauma Acute Care Surg 76 : 921-927; discussion 927-928, 2014 
9. Ghajar J : Traumatic brain injury. Lancet 356 : 923-929, 2000

10. Howard JT, Kotwal RS, Santos-Lazada AR, Martin MJ, Stockinger ZT : Reexamination of a battlefield trauma golden hour policy. J Trauma Acute Care Surg 84 : 11-18, 2018

11. Humphreys I, Wood RL, Phillips CJ, Macey S: The costs of traumatic brain injury: a literature review. Clinicoecon Outcomes Res 5 : 281287, 2013

12. Jennett $B$, Bond $M:$ Assessment of outcome after severe brain damage. Lancet $1:$ 480-484, 1975

13. Kane G, Wheeler NC, Cook S, Englehardt R, Pavey B, Green K, et al. : Impact of the Los Angeles county trauma system on the survival of seriously injured patients. J Trauma 32 : 576-583, 1992

14. Kim Y, Jung KY, Cho KH, Kim H, Ahn HC, Oh SH, et al. : Preventable trauma deaths rates and management errors in emergency medical system in Korea. J Korean Soc Emerg Med 17 : 385-394, 2006

15. Maio RF, Burney RE, Gregor MA, Baranski MG : A study of preventable trauma mortality in rural Michigan. J Trauma 41 : 83-90, 1996

16. Mann NC, Cahn RM, Mullins RJ, Brand DM, Jurkovich GJ : Survival among injured geriatric patients during construction of a statewide trauma system. J Trauma 50 : 1111-1116, 2001

17. Moon S, Lee SH, Ryoo HW, Kim JK, Ahn JY, Kim SJ, et al. : Preventable trauma death rate in Daegu, South Korea. Clin Exp Emerg Med 2 : 236-243, 2015

18. Mullins RJ, Veum-Stone J, Helfand M, Zimmer-Gembeck M, Hedges JR, Southard PA, et al. : Outcome of hospitalized injured patients after institution of a trauma system in an urban area. JAMA 271 : 1919-1924, 1994

19. Newgard CD, Meier EN, Bulger EM, Buick J, Sheehan K, Lin S, et al. : Revisiting the "Golden Hour": an evaluation of out-of-hospital time in shock and traumatic brain injury. Ann Emerg Med 66 : 30-41, 41.e1e3, 2015

20. Palmer $C$ : Major trauma and the injury severity score--where should we set the bar? Annu Proc Assoc Adv Automot Med 51 : 13-29, 2007

21. Park JM : Outcomes of the support services for the establishment of regional level 1 trauma centers. J Korean Med Assoc 59 : 923-930, 2016

22. Ponsford J, Olver J, Ponsford M, Nelms R : Long-term adjustment of families following traumatic brain injury where comprehensive rehabilitation has been provided. Brain Inj 17 : 453-468, 2003

23. Saatman KE, Duhaime AC, Bullock R, Maas Al, Valadka A, Manley GT, et al. : Classification of traumatic brain injury for targeted therapies. J Neurotrauma $25: 719-738,2008$

24. Sampalis JS, Lavoie A, Boukas S, Tamim H, Nikolis A, Fréchette $P$, et al. : Trauma center designation: initial impact on trauma-related mortality. J Trauma 39 : 232-237; discussion 237-239, 1995

25. Schluter PJ : The Trauma and Injury Severity Score (TRISS) revised. Injury $42: 90-96,2011$

26. Teasdale $G$, Jennett $B$ : Assessment of coma and impaired consciousness. A practical scale. Lancet 2 : 81-84, 1974

27. Teixeira PG, Inaba K, Hadjizacharia P, Brown C, Salim A, Rhee P, et al. : Preventable or potentially preventable mortality at a mature trauma center. J Trauma 63 : 1338-1346; discussion 1346-1347, 2007

28. Ulvik $A$, Kvåle $R$, Wentzel-Larsen $T$, Flaatten $H$ : Multiple organ failure after trauma affects even long-term survival and functional status. Crit Care 11 : R95, 2007

29. Yoon HD : Background and progress of regional trauma center development. J Korean Med Assoc 59 : 919-922, 2016 